\title{
The wide utility of rabbits as models of human diseases
}

Pedro J. Esteves ${ }^{1,2,3}$, Joana Abrantes ${ }^{1}$, Hanna-Mari Baldauff', Lbachir BenMohamed ${ }^{5,6,7}$, Yuxing Chen ${ }^{8}$, Neil Christensen ${ }^{9}$, Javier González-Gallego ${ }^{10}$, Lorenzo Giacani ${ }^{11}$, Jiafen Hu${ }^{9}$, Gilla Kaplan ${ }^{12}$, Oliver T. Keppler ${ }^{4}$, Katherine L. Knight ${ }^{13}$, Xiang-Peng Kong ${ }^{14}$, Dennis K. Lanning ${ }^{13}$, Jacques Le Pendu' ${ }^{15}$, Ana Lemos de Matos ${ }^{16}$, Jia Liu ${ }^{17}$, Shuying Liu', Ana M. Lopes ${ }^{1,18}$, Shan Lu ${ }^{8}$, Sheila Lukehart ${ }^{11}$, Yukari C. Manabe ${ }^{19}$, Fabiana Neves ${ }^{1}$, Grant McFadden ${ }^{16}$, Ruimin Pan ${ }^{14}$, Xuwen Peng ${ }^{9}$, Patricia de Sousa-Pereira ${ }^{1,2,4}$, Ana Pinheiro ${ }^{1,13}$, Masmudur Rahman ${ }^{16}$, Natalie Ruvoën-Clouet ${ }^{15}$, Selvakumar Subbian ${ }^{20}$, Maria Jesús Tuñón ${ }^{10}$, Wessel van der Loo ${ }^{1}$, Michael Vaine ${ }^{8}$, Laura E. Via ${ }^{21,22}$, Shixia Wang ${ }^{8}$ and Rose Mage ${ }^{23}$

\begin{abstract}
Studies using the European rabbit Oryctolagus cuniculus contributed to elucidating numerous fundamental aspects of antibody structure and diversification mechanisms and continue to be valuable for the development and testing of therapeutic humanized polyclonal and monoclonal antibodies. Additionally, during the last two decades, the use of the European rabbit as an animal model has been increasingly extended to many human diseases. This review documents the continuing wide utility of the rabbit as a reliable disease model for development of therapeutics and vaccines and studies of the cellular and molecular mechanisms underlying many human diseases. Examples include syphilis, tuberculosis, HIV-AIDS, acute hepatic failure and diseases caused by noroviruses, ocular herpes, and papillomaviruses. The use of rabbits for vaccine development studies, which began with Louis Pasteur's rabies vaccine in 1881, continues today with targets that include the potentially blinding HSV-1 virus infection and HIV-AIDS. Additionally, two highly fatal viral diseases, rabbit hemorrhagic disease and myxomatosis, affect the European rabbit and provide unique models to understand co-evolution between a vertebrate host and viral pathogens.
\end{abstract}

\section{Introduction}

Small laboratory animals, such as mice, rats, guinea pigs, and European rabbits, have long been used as models to improve our understanding of several human maladies. The primary goal of developing animal models for research is to create an experimental system in which the conditions occurring in humans are phenocopied

\footnotetext{
Correspondence: Pedro J. Esteves (pjesteves@cibio.up.pt) or

Rose Mage (rmage@niaid.nih.gov)

${ }^{1} \mathrm{CIBIO}$, InBIO, Research Network in Biodiversity and Evolutionary Biology, Universidade do Porto, Campus de Vairão, Rua Padre Armando Quintas, 4485-661 Vairão, Portugal

${ }^{2}$ Departamento de Biologia, Faculdade de Ciências da Universidade do Porto, Rua do Campo Alegre, s/n, 4169-007 Porto, Portugal

Full list of author information is available at the end of the article.

This article has been updated.
}

as accurately as possible in the laboratory animal. The rabbit was the first animal model used in several immunological studies and was crucial, for example, for the development of the rabies vaccine by Louis Pasteur in $1881^{1}$. The pioneering studies of rabies and syphilis conducted in rabbits continued to advance our understanding of these and other infectious diseases. Furthermore, the study of rabbit immunoglobulins established much of what is known about the structure, function and regulated expression of antibodies [reviewed in refs. ${ }^{2-4}$. Although, rabbit was a major animal model used for the study of molecular immunology in the late 1980s, rabbits were increasingly replaced by rodents in the subsequent years ${ }^{5}$. Among the reasons for the increasing use of rodents, such as mice, instead of 
rabbits are reduced maintenance costs, small size, availability of inbred strains, ease of breeding, short reproductive cycle, high numbers of progeny, wide availability of commercial immunological reagents, and availability of many knockout (KO) and transgenic models ${ }^{6,7}$. However, rabbits have the advantage of an intermediate size between rodents and larger, more costly animal models, such as primates. The size of rabbits permits the ready sampling of blood and greater access to many cells and tissues from a single animal. Additionally, rabbits have a longer life span than that of rodents, and the immune system genes of rabbits are apparently more similar to those of the human immune system than are rodent genes $^{8-10}$. Rabbits are also carriers or reservoirs of several pathogens that can cause zoonotic diseases. Some studies in mice found a lack of disease symptoms mimicking those of human infection. Additionally, the low-success rates in the translation of findings from some mouse studies to human diseases suggest that other animal models, such as rabbit, may often be more appropriate $^{11,12}$. The rabbit is actively used as a laboratory model for several non-infectious conditions, including atherosclerosis ${ }^{13,14}$, intestinal immunity ${ }^{15}$, reproduction ${ }^{16}$, lupus $^{17}$, arthritis ${ }^{18}$, cancer $^{19}$, and Alzheimer's disease ${ }^{20}$.

The rabbit has also been increasingly used during the last two decades as a reliable animal model for many infectious diseases. In this essay, several examples, including viral, bacterial, and parasitic infectious diseases, are described in which rabbits provide a more reliable model for host-pathogen interactions than rodents for their human disease counterparts.

\section{Overview of the rabbit immune system, therapeutics, and co-evolution between host and viral pathogens}

The pre-immune antibody repertoire in young rabbits develops in two stages: first, with antibody heavy chain-encoding gene segments $\mathrm{V}$ (variable), D (diversity), and $J$ (joining) rearrangements in bone marrow and, second, with immunoglobulin (Ig) gene diversification in gut-associated lymphoid tissue (GALT) ${ }^{21,22}$. In the bone marrow, one $\mathrm{VH}$ gene segment, $\mathrm{VH} 1$, is preferentially used for $\mathrm{V}(\mathrm{D}) \mathrm{J}$ gene rearrangements, whereas numerous kappa or lambda variable gene segments are used in VJ gene rearrangements of the light chain. After leaving the bone marrow and migrating to GALT, the B cells undergo proliferation and diversify their Ig genes by somatic hypermutation and gene conversion. Specific commensal microbes drive this B-cell expansion and Ig gene diversification through a mechanism that is not yet fully elucidated. Understanding how commensal bacteria drive these processes will provide insight into the hostmicrobial interactions that shape the pre-immune antibody repertoire.
The rabbit mucosal IgA system is highly unusual. Thirteen IgA C $\alpha$ genes are found in rabbits ${ }^{23}$, in contrast to most mammals, which have only one (e.g., mice) or two (e.g., hominoids) IgA subclasses. Of the $13 \mathrm{C} \alpha$ genes, most have allelic forms, and 11 are expressed as different IgA subclasses in mucosal tissues. Concomitantly, rabbits have lost the IgA-specific FcR and FcalphaRI ${ }^{24}$, and the IgA rabbit genes have been evolving under strong positive selection ${ }^{24,25}$. How the rabbit mucosal immune system has adapted to the many IgA subclasses and which FcRs bind these IgA subclasses remain a mystery; studies of this system may open new therapeutic possibilities.

In addition to the diversification of rearranged heavy and light chain genes that occurs in the GALT of young rabbits during pre-immune repertoire development, rabbits respond to infections or experimental immunizations in peripheral sites, including the spleen and lymph nodes, by producing highly specific antibodies of high affinity through diversification of rearranged heavy and light chain genes via both gene conversion and somatic hypermutation. For this reason, rabbits are a major source of polyclonal and monoclonal antibodies (mAbs) used for research and therapeutics.

A fully rabbit polyclonal anti-thymocyte globulin, approved by the FDA in 1998, remains in use in immunosuppressed patients to arrest acute rejection of kidney transplants ${ }^{26}$. For non-immunosuppressed patients, human polyclonals produced in rabbits with humanized immunoglobulin genes are under development ${ }^{27}$. Humanized rabbit mAbs that are currently being developed and tested include anti-human CD40, anti-vascular endothelial growth factor (VEGF), and a smaller singlechain fragment anti-VEGF (Fv) mAb (Brolucizumab) ${ }^{28}$. Strategies for humanization of rabbit mAbs are reviewed in refs. ${ }^{28,29}$.

Additionally, rabbits have been used for studies of therapeutics destined for treatment of human inhalational anthrax. Tests of the human mAb Raxibacumab ${ }^{30}$ and the chimeric mouse/human mAb Obiltoxaximab were conducted in many rabbits and fewer monkeys before approval for treatment of inhalational anthrax under the United States Food and Drug Administration (FDA) animal efficacy rule. Animal-to-human dose translation for Obiltoxaximab was also investigated in rabbits [reviewed in ref. ${ }^{31}$ ].

Chemokines and their receptors play a crucial role during immune responses. Because of gene conversion with the CCR2 gene ${ }^{32,33}$, the CCR5 gene of the European rabbit underwent a change at the region corresponding to the CCL8 docking site in humans ${ }^{34,35}$. The evolutionary study of this CCR5 ligand in Lagomorphs indicated an adaptive pseudogenization process of the CCL8 gene $^{36,37}$. This makes the European rabbit a suitable model for 
studies of the pathways of receptor and ligand interaction during inflammation-driven pathogenicity.

The European rabbit is also a good model to understand the co-evolution between vertebrate hosts and viral pathogens. In the last 60 years, wild and domestic rabbit populations suffered a sharp decline due to hunting, habitat destruction and the emergence of two viral diseases, namely, myxomatosis in the 1950s and rabbit hemorrhagic disease (RHD) in the 1980s. These diseases led to a contraction of rabbit populations with serious economic and ecological consequences [reviewed in ref. $\left.{ }^{38}\right]$. In Australia, the dynamic observed between the genetic resistance of natural European rabbit populations against myxomatosis and the virulence grades of its causative agent, the myxoma virus (MYXV), is one of the best textbook examples of virus/host co-evolution [reviewed in refs. ${ }^{39,40}$ ]. RHD, caused by the rabbit hemorrhagic disease virus (RHDV), was responsible for a dramatic decline in natural rabbit populations at the end of the 1980s. Although the populations subsequently recovered, the emergence, in 2010, of a new RHDV variant, genetically and antigenically highly distinct from the previously described strains, led to increased mortalities ${ }^{41}$. The new variant remains highly lethal and has high levels of recombination ${ }^{42}$, which provides an opportunity to understand how the vertebrate host and the virus will mutually co-evolve.

\section{Myxomatosis and oncolytic activity}

The natural history and biomedical relevance of the European rabbit cannot be dissociated from one of the most preponderant infectious agents of the species, the myxoma virus (MYXV). MYXV is a rabbit-specific poxvirus (family Poxviridae, genus Leporipoxvirus), and similar to many other poxviruses, its genes can be divided into two classes: "essential" genes (i.e., required for virus propagation in culture) encoding proteins involved in viral replication, gene expression and virion assembly and "non-essential" genes (i.e., those that can be deleted with continued virus propagation in culture) encoding factors required for host-range, immunomodulation, and virulence $\mathrm{e}^{39,40}$. The host-range genes evolved in the natural Sylvilagus hosts of MYXV, the South American tapeti and the North American brush rabbit, in which the virus causes a localized cutaneous fibroma. However, when MYXV infected the naive European rabbit as a new host, the virus caused an outbreak of the novel and lethal systemic disease myxomatosis ${ }^{39,40}$.

The study of MYXV and European rabbit interaction makes an important contribution to the field of emerging infectious diseases. It provides an outstanding model to study dynamic host-pathogen interactions and makes myxomatosis in the European rabbit an exceptional example of host-virus co-evolution. The selective tropism of MYXV toward the European rabbit was fundamental to studies of the MYXV host-range and immunomodulatory genes and their encoded proteins, which continue to evolve in the new host. Targeted knockouts of these viral genes frequently resulted in virus attenuation in the rabbit host and consequently allowed a deeper understanding of the molecular basis for MYXV pathogenesis in the European rabbit ${ }^{39}$. Such knowledge has also been important for the ongoing development of MYXV as a potential oncolytic virotherapeutic for the treatment of a variety of human cancers by exploiting the ability of the virus to productively infect a wide diversity of non-rabbit cancer cells ${ }^{43,44}$. For example, the M135R-knockout MYXV cannot induce myxomatosis in European rabbits and is non-pathogenic for all known vertebrate hosts; however, this knockout is fully oncolytic in human cancer cells and is currently being developed as a clinical candidate for oncolytic immunotherapy to treat human hematological malignancies ${ }^{44,45}$.

\section{Rabbit hemorrhagic disease virus as a model of human noroviruses and hepatic fulminant diseases}

Initial studies on RHDV showed that it attached to glycans of the histo-blood group antigens (HBGAs) ${ }^{46}$. Because this virus belongs to the family Caliciviridae, these results provided the impetus to search for glycans potentially used by human caliciviruses, such as noroviruses, which constitute a major cause of gastroenteritis worldwide $^{47}$. Unlike mouse strains, human strains of noroviruses, although also attaching to HBGAs, do not all present the same pattern of recognition ${ }^{48}$. Because of the genetic polymorphism of HBGAs, not all humans are equally susceptible to individual strains of noroviruses, suggesting a past and possibly ongoing co-evolution between humans and noroviruses ${ }^{49}$. Using the European rabbit as a model, a survey of the frequency of their HBGA polymorphisms in wild populations affected by the virus conducted in parallel with a survey of the evolution of the specificity for HBGA recognition of the virus itself allowed documentation of the co-evolution between host and pathogen at a molecular level, underscoring the importance of the rabbit as a model for relevant human gastrointestinal pathogens ${ }^{50,51}$.

Acute hepatic failure (AHF) is a severe liver injury accompanied by encephalopathy that causes multi-organ failure with an extremely high-mortality rate. Severe AHF continues to be one of the most challenging problems in clinical medicine. Treatment has been limited by the lack of satisfactory animal models, particularly for acute viral hepatitis, a frequent cause of this condition. RHDV inoculation in rabbits is an excellent model for AHF of viral origin, displaying biochemical/histological characteristics, presence of encephalopathy, and clinical 
features that resemble those in humans ${ }^{52}$. Additional advantages in comparison with rodent models are the larger size of rabbits and the sufficient time window before death, which renders testing of new liver support systems possible and permits sufficient samples of blood and tissue to be taken during treatments. Increased insight was gained into the physiologic derangements of virus-induced $\mathrm{AHF}^{53}$ using the RHDV model, and beneficial effects of experimental treatment with different $\operatorname{drugs}^{54,55}$ and antioxidants ${ }^{56-58}$ were identified. The emerging new RHDV2 variant, which differs from RHDV in terms of duration of induced disease, mortality rates and higher occurrence of subacute/chronic forms ${ }^{59}$, opens the possibility to establish rabbit models for chronic liver diseases.

\section{Syphilis}

Since, the identification and isolation of Treponema pallidum subsp. pallidum (T. pallidum) as the causative agent of syphilis ${ }^{60}$, the rabbit has been the model of choice for the study of the infection and for propagation of this uncultivable bacterium. Rabbits can be readily infected with $T$. pallidum ${ }^{61}$, which is perhaps related to their susceptibility in nature to a very closely related bacterium, Treponema paraluiscuniculi, which is sexually transmitted. The clinical, histological, and immunological similarities between syphilis infection in rabbit and human hosts are striking. Intradermal inoculation of rabbits with $T$. pallidum results in the development of lesions that strongly resemble human primary chancres both clinically and histologically ${ }^{62,63}$, with CD4+ and CD8+ $\mathrm{T}$ lymphocytes and macrophage infiltration. As observed in humans, the primary chancres in rabbits resolve spontaneously, with subsequent development of a disseminated secondary stage rash and early invasion of the central nervous system. Rabbits mount an immune response similar to that developed by humans during natural infection ${ }^{63}$, with recognition of the same subset of antigens and the same mechanisms of bacterial clearance. As in humans, following long-term infection, rabbits develop immunity to reinfection ${ }^{62}$. Rabbit size facilitates the study of dissemination of the bacteria to distant tissue sites $^{64}$, and rabbits provide models of congenital and neurosyphilis ${ }^{65-67}$. Because T. pallidum cannot be cultured, rabbits are also critical for the isolation of new T. pallidum strains from clinical samples. Although some other animal species can be infected with T. pallidum (non-human primates [NHP], hamsters, guinea pigs, and mice ${ }^{62,68-70}$ ), only rabbits and NHP develop clinical disease similar to that in humans. Despite the lack of inbred rabbit strains and the dearth of immunological reagents, the rabbit model continues to be the most widely used by syphilis investigators to deepen our understanding of syphilis pathogenesis ${ }^{71}$, evaluate the efficacy of new therapies ${ }^{72}$, and test the protective ability of novel vaccine candidates $^{73}$.

\section{Tuberculosis}

Rabbits were used in Robert Koch's original experiments to establish Mycobacterium tuberculosis (MTB) as the causative agent of human tuberculosis $(\mathrm{TB})^{74}$ and extensively utilized thereafter. Experiments of both MTB infection, to which rabbits are relatively resistant, and Mycobacterium bovis (MBO) infection, which is much more virulent in rabbits, were pursued in great detail by Lurie in a cohort of 'resistant' and 'susceptible' rabbits ${ }^{75}$. These seminal studies laid the foundation for future research on the host genetic predisposition to mycobacterial infections. The New Zealand White (NZW) partially inbred European rabbit strain developed by J. Thorbecke was also susceptible to MTB infection ${ }^{76}$; this breed was used for the rabbit genome sequencing project (OryCun2.0). After the unexpected extinction of these rabbits, outbred New Zealand White rabbits were the most commonly used breed for TB research to model the human pathology of pulmonary active/cavitary TB (PTB), non-progressive latent MTB infection (LTBI), spinal TB and tuberculous meningitis $(\mathrm{TBM})^{77-81}$. Intrathecal or intracisternal infections of rabbits with virulent MTB or $\mathrm{MBO}$ cause progressive disease pathology in the brain, ultimately resulting in encephalopathy and paralysis characteristic of $\mathrm{TBM}^{78,82}$.

The primary advantage of rabbit models over the mouse model of TB is the maturation of inflammatory leukocytic foci into organized granulomas following aerosol infection. These lesions often undergo caseating necrosis, the pathologic hallmark of TB, and can develop fibrosis and/ or cavitation, in addition to mineralization depending on the bacterial strain used for infection ${ }^{77,81,83,84}$. In contrast to most mouse models, necrotic granulomas in rabbits also develop hypoxic microenvironments ${ }^{85}$, which can serve as models for drug exposure studies.

The outcome of pulmonary infection in outbred NZW rabbits is also dependent on the nature of the infecting MTB strain. Whereas infection with hypervirulent MTB strains of the W-Beijing lineage cause cavitary $\mathrm{TB}$, other strains, such as the hyperimmunogenic CDC1551, cause a range of disease presentation, including LTBI, which can reactivate upon immune suppression treatment ${ }^{81,86}$. The ability of rabbits to control MTB infection, establish LTBI and reactivate to active disease upon immunosuppression provides a unique model for studies of the regulation of latency, reactivation, and immune reconstitution syndromes similar to those observed in human immunodeficiency virus infection ${ }^{77,79,81}$.

The rabbit model has helped to elucidate the pharmacologic properties of standard and new/novel anti-MTB compounds, demonstrating a similar drug distribution 
and pharmacokinetic/pharmacodynamic properties as in studies in humans undergoing lung resection surgery ${ }^{87-91}$. Immune-modulating host-directed adjunctive therapy has emerged as a novel approach to improve TB treatment. Several proof-of-concept studies conducted in rabbit models of PTB and TBM demonstrated that these drugs not only improve bacterial killing but also minimize the disease pathology and restore organ and vasculature function $^{92,93}$. Rabbit models have also been used to test the efficacy of TB vaccines, including BCG, M. vaccae, M. microti and MTB fusion proteins in protecting against MTB challenge ${ }^{82,94,95}$.

In summary, the rabbit model of $\mathrm{TB}$ has great histopathologic similarity to human disease. Therefore, this model will continue to play a vital role in deciphering the intricate pathogenesis of various forms of human TB and in devising better intervention strategies.

\section{Human papillomaviruses (HPV)}

HPV are important viral pathogens that cause a variety of mucosal infections of the anogenital and oral mucosa. These infections can lead to epithelial cancers at these mucosal sites. Papillomaviruses (PVs) are speciesrestricted such that HPVs cannot be directly studied in pre-clinical laboratory animal models. Two rabbit papillomavirus models have been used extensively to study various aspects of papillomavirus biology, including vaccine testing ${ }^{96}$, anti-viral treatments ${ }^{97}$, papillomavirus biology $^{98}$, and latent viral infections ${ }^{99}$. The viruses include cottontail rabbit papillomavirus (CRPV), which is a cutaneous-tropic virus whose lesions spontaneously progress to cancer, and rabbit oral papillomavirus (ROPV), which is a mucosa-tropic virus that induces oral infections. Numerous viral mutant genomes ${ }^{98}$ and a unique HLA.A2.1 transgenic rabbit line ${ }^{100}$ have been developed to study host immune responses to viral infection, therapeutic T-cell-based vaccines and host anti-CD8 immunity to viral proteins.

\section{Human immunodeficiency virus (HIV)}

Human immunodeficiency virus (HIV-1) is the causative agent of AIDS. The development of a permissive, readily available, immunocompetent small animal model for the study of HIV-1 transmission and pathogenesis and the testing of antiviral strategies has been hampered by the inability of HIV-1 to infect non-human cells productively. Over the last 20 years, multiple species-specific barriers to HIV-1 replication have been identified in mouse, rat, and rabbit cells ${ }^{101-107}$, with some caused by non-functional cellular cofactors or potent antiviral restriction factors that directly target the incoming virus (e.g., HIV-1 capsid by rabbit TRIM5-alpha ${ }^{106}$ ) or cannot be antagonized by the accessory proteins of HIV-1 (e.g., rat and mouse CD317/tetherin by HIV-1 $\mathrm{Vpu}^{108}$ or rabbit APOBEC1 by HIV-1 Vif ${ }^{109}$ ). This knowledge has fueled strategies to generate genetically modified small animals in which transgenesis, knock-in, and knockout approaches combined with limited modifications to HIV-1 may allow the virus to overcome species-specific limitations and render the rodent or lagomorph host fully permissive to infection by this pathogenic human lentivirus. In contrast to several thus far unresolved replication defects in the late phase in mice ${ }^{110}$ and, to a lesser extent, in rats ${ }^{104}$, primary $\mathrm{T}$ cells and macrophages from rabbits impose only three apparent barriers to HIV1 replication: virion entry, which can be overcome by coexpression of the HIV-1 receptor complex composed of human CD4 and CCR $5^{107,111}$; reverse transcription, which can be ameliorated by depletion of TRIM5-alpha or modifications of HIV-1 gag; and a cell type-specific infectivity defect of HIV-1 virions released from macrophages, the cause of which is still unclear ${ }^{107}$. This knowledge combined with recent advances in knock-in and knockout technologies ${ }^{12,113}$ and the overall suitability for vaccine and drug studies makes the rabbit species an attractive candidate for the generation of a fully permissive animal model of HIV-1 infection.

Additionally, the rabbit model has been used extensively in developing an HIV vaccine. In contrast to other small animal models, such as mouse or rat, the rabbit model provides several advantages that include ease of induction of high-titer, high-affinity antigen-specific antibody responses to almost any type of antigen and very low non-specific responses. Consequently, rabbit immune sera can be used for a wide range of assays, including ELISA, western blots, and tests for functional antibody responses such as neutralizing antibodies ${ }^{114-120}$. The rabbit model was used first to study polyclonal antibody responses and established the immunogenicity of DNA immunization as a novel immunization method ${ }^{121}$. Subsequently, a DNA prime-protein boost approach was developed $^{122}$, and it was established that this approach elicited neutralizing antibodies against a difficult virus ${ }^{123}$, improved induction of antibodies against key neutralizing epitopes, and improved activity and avidity ${ }^{124-126}$. More recently, a panel of novel rabbit monoclonal antibodies (mAbs) against HIV-1 Env antigen was produced, and the crystal structures for some of these rabbit mAbs were obtained (Fig. 1) ${ }^{127-129}$. These studies also demonstrated that the overall structures of rabbit mAbs are very similar to those of human mAbs against the same epitopes. This is highly significant considering that the mechanisms to diversify these two types of mAbs are very different. Overall, rabbits have been an excellent animal model to study the immunogenicity of HIV-1 DNA vaccines, including the production and analysis of HIV-1 specific $\mathrm{mAbs}^{130}$. 


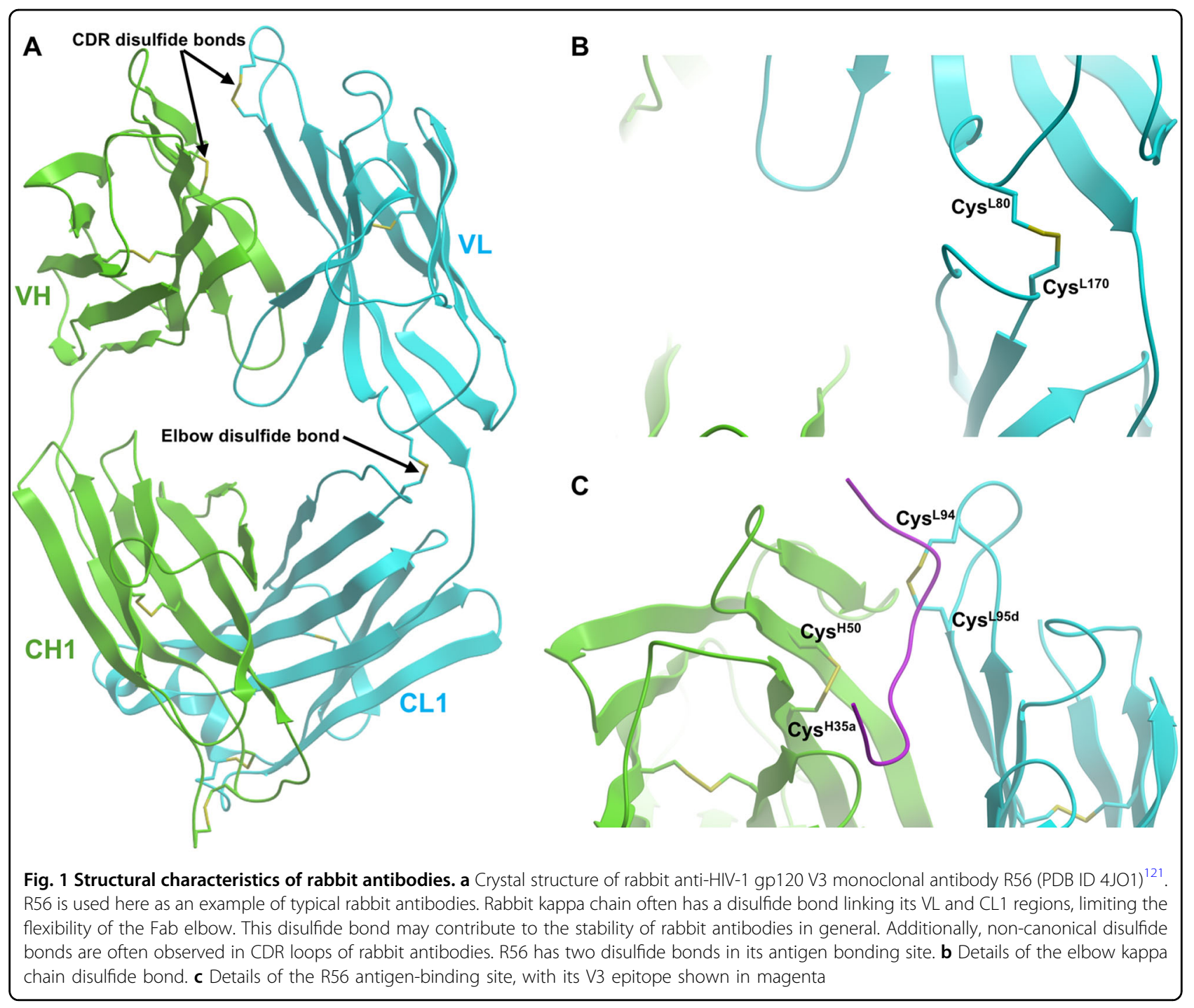

\section{Ocular herpes infection and immunity}

Most of the potentially blinding, recurrent herpes stromal keratitis (rHSK) in latently infected humans occurs following spontaneous reactivation of HSV-1 from latently infected sensory neurons and virus shedding in tear film ${ }^{131-135}$. However, unlike in latently infected humans, spontaneous HSV-1 reactivation and virus shedding in tears occurs at very low levels or not at all in latently infected mice ${ }^{132,135,136}$. Despite this fact, most pre-clinical animal studies investigating the cellular and molecular mechanisms that orchestrate rHSK have used a mouse model of primary acute infection ${ }^{136}$. To avoid inherent drawbacks in the mouse model of primary acute infection, an alternative HLA transgenic rabbit (HLA Tg rabbit) model in which HSV-1 reactivation and virus shedding in tear film occur spontaneously was developed $^{137}$. One major component of the immune system in these HLA $\mathrm{Tg}$ rabbits is replaced by the identical component taken from a human counterpart (i.e., HLAA*0201 class I molecules) ${ }^{137-143}$. This HLA Tg rabbit model is capable of mounting "human-like" $\mathrm{CD} 8^{+} \mathrm{T}$-cell responses specific to human HLA-A*0201-restricted epitopes. The immunopathology of rHSK in the HLA Tg rabbit mimics the immunopathology of human rHSK that occurs after episodes of spontaneous HSV-1 reactivation (Fig. 2). Moreover, this HLA Tg rabbit model allows preclinical investigation of the role of HLA-restricted CD8 ${ }^{+}$ $\mathrm{T}$-cell responses specific to human epitopes in reducing spontaneous reactivation of $\mathrm{HSV}-1$, and assessment of the immunotherapeutic efficacy of human $\mathrm{CD}^{+}{ }^{+}$T-cell epitope-based vaccines against rHSK $^{137-143}$. Recently developed immunological reagents (e.g., mAbs specific for immune molecules and human tetramers) have allowed analysis of rabbit HSV-specific $\mathrm{CD}^{+} \mathrm{T}$ cells' phenotype, function, and the localization of $\mathrm{CD}^{+}{ }^{+} \mathrm{T}$-cell infiltrates in infected cornea and trigeminal ganglia 


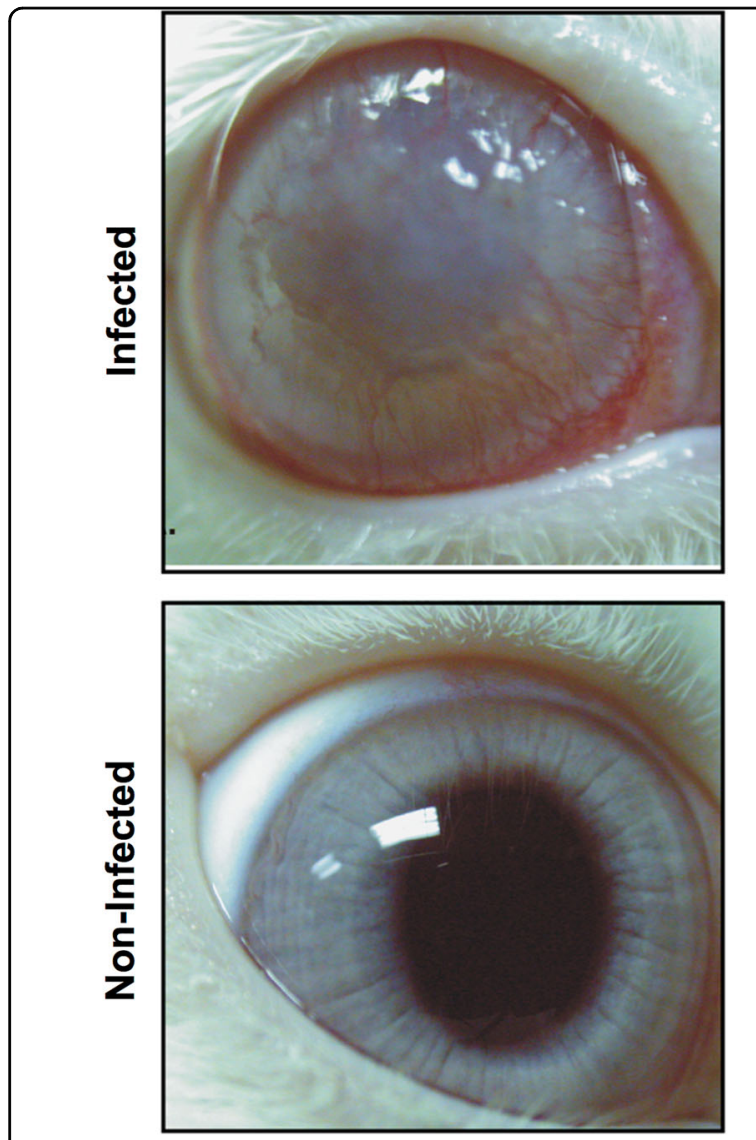

Fig. 2 Infected and non-infected rabbit eyes. Eyes of 8-week-old New Zealand White (NZW) female rabbits on day 35 after ocular infection with HSV-1 $\left(2 \times 10^{5} \mathrm{pfu} /\right.$ eye of strain McKrae). Eye pictures were taken under regular light

(the site of latency/reactivation cycles) of infected HLA Tg rabbits $^{130}$. The striking similarities between the HSV-1infected HLA Tg rabbit model and HSV-1 seropositive humans in terms of ocular herpes infection and immunity make the HLA Tg rabbit a preferred model to study the role of $\mathrm{CD}^{+} \mathrm{T}$ cells in controlling spontaneous HSV-1 reactivation and recurrent $\mathrm{HSK}^{130-137}$.

\section{Conclusions}

Studies using the European rabbit contributed to elucidating numerous fundamental aspects of antibody structure and diversification mechanisms and continue to be valuable for the development and testing of therapeutics. Rabbits have also served as important, reliable models for the understanding of human infectious and non-infectious diseases, including tuberculosis, syphilis, and papilloma-, herpes-, pox-, and norovirus infections, and have been adapted to advance the understanding of the immune response to HSV-1 and HIV-1 to inform immunotherapy and vaccine development.

\section{Acknowledgements}

This research was supported in part by the Intramural Research Program of the NIH, NIAID (R.M. and L.E.V.), in part by Bill and Melinda Gates Foundation grants OPP1034408 and OPP1 140482 and in part by NIH grants Al082274 (S.L.), Al100151 (X.P.K.), Al50260 and Al068390 (K.L.K. and D.L.), and R03Al119619 (S.S.). J.L. is supported in part by K22-A99184, P20GM103625, the Cookie Laughlin Award from the Rivkin Center for Ovarian Cancer, and a start-up fund by the UAMS Department of Microbiology and Immunology. L.B. is supported by the Public Health Service Research R01 Grant EY019896 and by the National Eye Institute (NEI). N.D.C. and J.H. are supported by NIH grant 5R01 CA47622 by the National Cancer Institute. H.-M.B. is supported by LMU Munich's Institutional Strategy LMU excellent within the framework of the German Excellence Initiative. CIBERehd (J.G.-g and M.J.T.) is funded by the Instituto de Salud Carlos III, Spain. The Portuguese Foundation for Science and Technology (FCT) supported the FCT Investigator grants of J.A. (ref.: IF/01396/ 2013) and P.J.E. (ref.: IF/00376/2015), the Post-Doc grants of A.M.L. (ref.: SFRH/ BPD/115211/2016) and A.P. (ref.: SFRH/BPD/117451/2016) and the PhD grants of P.d.S.P. (ref.: PD/BD/52602/2014) and F.N. (SFRH/BD/81916/2011). J.L.P. is funded by Inserm.

\section{Author details}

${ }^{1} \mathrm{CIBIO}, \ln \mathrm{BIO}$, Research Network in Biodiversity and Evolutionary Biology, Universidade do Porto, Campus de Vairão, Rua Padre Armando Quintas, 4485-661 Vairão, Portugal. ²Departamento de Biologia, Faculdade de Ciências da Universidade do Porto, Rua do Campo Alegre, s/n, 4169-007 Porto, Portugal. ${ }^{3}$ Instituto de Investigação e Formação Avançada em Ciências e Tecnologias da Saúde (CESPU), Gandra, Portugal. ${ }^{4}$ Max von Pettenkofer Institute and Gene Center, Virology, National Reference Center for Retroviruses, Faculty of Medicine, LMU München, 81377 Munich, Germany. ${ }^{5}$ Laboratory of Cellular and Molecular Immunology, Gavin Herbert Eye Institute, University of California, Irvine, School of Medicine, Irvine, CA 92697, USA. ${ }^{6}$ Department of Molecular Biology and Biochemistry, University of California, Irvine School of Medicine, Irvine, CA 92697, USA. ${ }^{7}$ Institute for Immunology, University of California, Irvine School of Irvine, School of Medicine, Irvine, CA 92697, USA. ${ }^{8}$ Department of Medicine, University of Massachusetts Medical School, Worcester, MA 01605, USA. ${ }^{9}$ Departments of Pathology, Microbiology and Immunology, and Comparative Medicine, Penn State University, Hershey, PA, USA. ${ }^{10}$ Institute of Biomedicine (IBIOMED) and Centro de Investigación Biomédica en Red de Enfermedades Hepáticas y Digestivas (CIBERehd), University of León, 24071 León, Spain. ${ }^{11}$ Departments of Medicine and Global Health, University of Washington, Seattle, USA. ${ }^{12}$ Bill and Melinda Gates Foundation, Seattle, WA, USA. ${ }^{13}$ Department of Microbiology and Immunology, Loyola University Chicago, Maywood, IL 60153, USA. ${ }^{14}$ Department of Biochemistry and Molecular Pharmacology, New York University School of Medicine, New York NY10016, USA. ${ }^{15}$ CRCINA, Inserm, Université d'Angers, Université de Nantes, Nantes, France. ${ }^{16}$ The Biodesign Institute, Center for Immunotherapy, Vaccines, and Virotherapy, Arizona State University, Tempe, AZ 85287-5401, USA. ${ }^{17}$ Department of Microbiology and Immunology, University of Arkansas for Medical Sciences (UAMS), Little Rock, AR 72205, USA. ${ }^{18}$ Department of Anatomy and Unit for Multidisciplinary Research in Biomedicine (UMIB), Institute of Biomedical Sciences Abel Salazar (ICBAS), University of Porto, Porto, Portugal. ${ }^{19}$ Division of Infectious Diseases, Department of Medicine, Johns Hopkins University School of Medicine, Baltimore, MD, USA. ${ }^{20}$ The Public Health Research Institute (PHRI) at New Jersey Medical School, Rutgers Biomedical and Health Sciences (RBHS), Rutgers University, Newark, NJ, USA. ${ }^{21}$ Tubercolosis Research Section, Laboratory of Clinical Infectious Diseases, Division of Intramural Research, National Institute of Allergy and Infectious Diseases, National Institutes of Health, Bethesda, MD, USA. ${ }^{22}$ Institute of Infectious Disease and Molecular Medicine, Department of Clinical Laboratory Sciences, University of Cape Town, Cape Town, South Africa. ${ }^{23}$ Laboratory of Immune System Biology, National Institute of Allergy and Infectious Diseases, National Institutes of Health, Bethesda, MD, USA

\section{Conflict of interest}

The authors declare that they have no conflict of interest.

\section{Publisher's note}

Springer Nature remains neutral with regard to jurisdictional claims in published maps and institutional affiliations. 
Received: 28 November 2017 Revised: 21 February 2018 Accepted: 27 February 2018

Published online: 22 May 2018

\section{References}

1. Pasteur, L. Méthode pour prévenir la rage après morsure. Comptes rendus Hebd. Des. séances De. l'Académie Des. Sci. 101, 765-774 (1885).

2. Pinheiro, A. et al. Molecular bases of genetic diversity and evolution of the immunoglobulin heavy chain variable region (IGHV) gene locus in leporids. Immunogenetics 63, 397-408 (2011).

3. Pinheiro, A. et al. An overview of the lagomorph immune system and its genetic diversity. Immunogenetics 68, 83-107 (2016).

4. Mage, R. G., Pinheiro, A., Lemos de Matos, A. \& Esteves, P. J. The immune system of lagomorphs. In: M. J. H. Ratcliffe (ed). Encyclopedia of immunobiology. Vol. 1, (515-525. Elsevier, Amsterdam, Holand, 2016).

5. Burkholder, T. H., Linton, G. F. R., Hoyt, J. \& Young, R. The rabbit as an experimental model. In: M. A. Suckow, K. A. Stevens, R. P. Wilson (eds). The laboratory rabbit, guinea pig, hamster, and other rodents. pp. 529-560. (Academic Press, Cambridge, MA, USA, 2012).

6. Mullane, K. \& Williams, M. Animal models of asthma: reprise or reboot? Biochem. Pharmacol. 87, 131-139 (2014).

7. Webb, D. R. Animal models of human disease: inflammation. Biochem. Pharmacol. 87, 121-130 (2014).

8. Graur, D., Duret, L. \& Gouy, M. Phylogenetic position of the order Lagomorpha (rabbits, hares and allies). Nature 379, 333-335 (1996).

9. Neves, F. et al. Genetic characterization of interleukins (IL-1alpha, IL-1beta, IL-2, IL-4, IL-8, IL-10, IL-12A, IL-12B, IL-15 and IL-18) with relevant biological roles in lagomorphs. Innate Immun. 21, 787-801 (2015).

10. Perkins, H. D., van Leeuwen, B. H., Hardy, C. M. \& Kerr, P. J. The complete CDNA sequences of $I L-2,\|L-4\| L-$,6 AND IL-10 from the European rabbit (Oryctolagus cuniculus). Cytokine 12, 555-565 (2000).

11. James, J. et al. Forced expression of alpha-myosin heavy chain in the rabbit ventricle results in cardioprotection under cardiomyopathic conditions. Circulation 111, 2339-2346 (2005).

12. Zschaler, J., Schlorke, D. \& Arnhold, J. Differences in innate immune response between man and mouse. Crit. Rev. Immunol. 34, 433-454 (2014).

13. Fan, J. et al. Rabbit models for the study of human atherosclerosis: from pathophysiological mechanisms to translational medicine. Pharmacol. Ther. 146, 104-119 (2015).

14. Tian, J. et al. A novel model of atherosclerosis in rabbits using injury to arterial walls induced by ferric chloride as evaluated by optical coherence tomography as well as intravascular ultrasound and histology. J. Biomed. Biotechnol. 2014, 121867 (2012).

15. Jiménez-García, A. et al. Intestinal wall damage in simple ileus in rabbits: immune-modulator role of somatostatin. Hepatogastroenterology $\mathbf{5 1}$, 1030-1036 (2004).

16. Fischer, B., Chavatte-Palmer, P., Viebahn, C., Navarrete Santos, A. \& Duranthon, $\checkmark$. Rabbit as a reproductive model for human health. Reproduction 144, 1-10 (2012).

17. Mage, R. G. \& Rai, G. A rabbit model of systemic lupus erythematosus, useful for studies of neuropsychiatric SLE. In: H. Almoallin (ed). Systemic Lupus Erythematosus. pp. 201-217. (InTech -Open Access Publisher, Rijeka, Croatia, 2012).

18. Desando, G. et al. Intra-articular delivery of adipose derived stromal cells attenuates osteoarthritis progression in an experimental rabbit model. Arthritis Res. Ther. 15, R22 (2013).

19. Kang, S. J. \& Grossniklaus, H. E. Rabbit model of retinoblastoma. J. Biomed. Biotechnol. 2011, 394730 (2011).

20. Woodruff-Pak, D. S., Agelan, A. \& Del Valle, L. A rabbit model of Alzheimer's disease: valid at neuropathological, cognitive, and therapeutic levels. J. Alzheimers Dis. 11, 371-383 (2007).

21. Rhee, K. J. et al. Positive selection of the peripheral $B$ cell repertoire in gutassociated lymphoid tissue. J. Exp. Med. 201, 55-62 (2005).

22. Mage, R. G., Lanning, D. \& Knight, K. L. B cell and antibody repertoire development in rabbits: the requirement of gut-associated lymphoid tissues. Dev. Comp. Immunol. 30, 137-153 (2006).

23. Burnett, R. C., Hanly, W. C., Zhai, S. K. \& Knight, K. L. The IgA heavy-chain gene family in rabbit: cloning and sequence analysis of $13 \mathrm{C}$ alpha genes. EMBO J. 8, 4041-4047 (1989).
24. Abi-Rached, L., Dorighi, K., Norman, P. J., Yawata, M. \& Parham, P. Episodes of natural selection shaped the interactions of IgA-Fc with FcalphaRI and bacterial decoy proteins. J. Immunol. 178, 7943-7954 (2007).

25. Pinheiro, A., Woof, J. M., Abi-Rached, L., Parham, P. \& Esteves, P. J. Computational analyses of an evolutionary arms race between mammalian immunity mediated by immunoglobulin $\mathrm{A}$ and its subversion by bacterial pathogens. PLOS ONE 8, e73934 (2013).

26. Deeks, E. D. \& Keating, G. M. Rabbit anti-thymocyte globulin (thymoglobulin): a review of its use in the prevention and treatment of acute renal allograft rejection. Drugs 69, 1483-1512 (2009).

27. Flisikowska, T. et al. Efficient immunoglobulin gene disruption and targeted replacement in rabbit using zinc finger nucleases. PLOS ONE 6, e21045 (2011).

28. Weber, J., Peng, H. \& Rader, C. From rabbit antibody repertoires to rabbit monoclonal antibodies. Exp. Mol. Med. 49, e305 (2017).

29. Zhang, Y. F. \& Ho, M. Humanization of rabbit monoclonal antibodies via grafting combined Kabat/MGT/Paratome complementarity-determining regions: rationale and examples. MAbs 9, 419-429 (2017).

30. Kummerfeldt, C. E. Raxibacumab: potential role in the treatment of inhalational anthrax. Infect. Drug Resist. 7, 101-109 (2014).

31. Greig, S. L. Obiltoxaximab: first global approval. Drugs 76, $823-830$ (2016).

32. Carmo, C., Esteves, P. J., Ferrand, N. \& van der Loo, W. Genetic variation at chemokine receptor CCR5 in leporids: alteration at the 2 nd extra cellular domain by gene conversion with CCR2 in Oryctolagus, but not in Sylvilagus and Lepus species. Immunogenetics 58, 494-501 (2006).

33. Abrantes, J. et al. A shared unusual genetic change at the chemokine receptor type 5 between Oryctolagus, Bunolagus and Pentalagus. Conserv. Genet 12, 325-330 (2011).

34. Lau, G., Labrecque, J., Metz, M., Vaz, R. \& Fricker, S. P. Specificity for a CCR5 inhibitor is conferred by a single amino acid residue ROLE OF ILE198. J. Biol. Chem. 290, 11041-11051 (2015).

35. Proost, $\mathrm{P}$. et al. Posttranslational modifications affect the activity of the human monocyte chemotactic proteins MCP-1 and MCP-2: identification of MCP-2 (6-76) as a natural chemokine inhibitor. J. Immunol. 160, 4034-4041 (1998).

36. van der Loo, W., Afonso, S., de Matos, A. L., Abrantes, J. \& Esteves, P. J. Pseudogenization of the MCP 2/CCL8 Chemokine Gene in European Rabbit (genus Oryctolagus), but not in species of Cottontail Rabbit (Sylvilagus) and Hare (Lepus). BMC Genet. 13, 72 (2012).

37. van der Loo, W. et al. Adaptive gene loss? Tracing back the pseudogenization of the Rabbit CCL8 chemokine. J. Mol. Evolution 83, 12-25 (2016).

38. Abrantes, J., van der Loo, W., Le Pendu, J. \& Esteves, P. J. Rabbit haemorrhagic disease $(\mathrm{RHD})$ and rabbit haemorrhagic disease virus (RHDV): a review. Vet. Res. 43, 12 (2012).

39. Kerr, P. J. Myxomatosis in Australia and Europe: a model for emerging infectious diseases. Antivir. Res. 93, 387-415 (2012).

40. Kerr, P. J. et al. Myxoma virus and the Leporipoxviruses: an evolutionary paradigm. Viruses 7, 1020-1061 (2015).

41. Le Gall-Reculé, G. et al. Detection of a new variant of rabbit haemorrhagic disease virus in France. Vet. Rec. 168, 137-138 (2011).

42. Lopes, A. M. et al. Full genomic analysis of new variant Rabbit Hemorrhagic Disease Virus (RHDVb) revealed multiple recombination events. J. Gen. Virol. 96, 1309-1319 (2015).

43. Chan, W. M. et al. Myxoma and vaccinia viruses bind differentially to human leukocytes. J. Virol. 87, 4445-4460 (2013).

44. Chan, W. M. \& McFadden, G. Oncolytic poxviruses. Annu Rev. Virol. 1, 119-141 (2014).

45. Lilly, C. L. et al. Ex-vivo oncolytic virotherapy with myxoma virus arms multiple allogeneic bone marrow transplant leukocytes to enhance graft versus tumor. Mol. Ther. 4, 31-40 (2017).

46. Ruvoën-Clouet, N., Ganière, J. P., André-Fontaine, G., Blanchard, D. \& Le Pendu, J. Binding of rabbit hemorrhagic disease vrus to antigens of the $\mathrm{ABH}$ histo-blood group family. J. Virol. 74, 11950-11954 (2000).

47. Ahmed, S. M. et al. Global prevalence of norovirus in cases of gastroenteritis: a systematic review and meta-analysis. Lancet Infect. Dis. 14, 725-730 (2014).

48. Tan, M. \& Jiang, X. Histo-blood group antigens: a common nich for norovirus and rotavirus. Expert. Rev. Mol. Med. 16, e5 (2014).

49. Ruvöen-Clouet, N., Belliot, G. \& Le Pendu, J. Noroviruses and histo-blood groups: the impact of common host genetic polymorphisms on virus transmission and evolution. Rev. Med. Virol. 23, 355-366 (2013).

50. Le Pendu, J., Nystrom, K. \& Ruvoen-Clouet, N. Host-pathogen co-evolution and glycan interactions. Curr. Opin. Virol. 7, 88-94 (2014). 
51. Nyström, K. et al. Histo-blood group antigens act as attachment factors of rabbit hemorrhagic disease virus infection in a virus strain-dependent manner. PLoS Pathog. 7, e1002188 (2011).

52. Tuñón, M. J., Alvarez, M., Culebras, J. M. \& González-Gallego, J. An overview of animal models for investigating the pathogenesis and therapeutic strategies in acute hepatic failure. World J. Gastroenterol. 15, 3086-3098 (2009).

53. Vallejo, D. et al. Autophagic response in the rabbit hemorrhagic disease, an animal model of virally-induced fulminant hepatic failure. Vet. Res. 45, 15 (2014).

54. Tuñón, M. J. et al. Cardiotrophin-1 promotes a high survival rate in rabbits with lethal fulminant hepatitis of viral origin. J. Virol. 85, 13124-13132 (2011).

55. Booth, L. et al. AR-12 inhibits multiple chaperones concomitant with stimulating autophagosome formation collectively preventing virus replication. J. Cell Physiol. 231, 2286-2302 (2016).

56. San-Miguel, B., Alvarez, M., Culebras, J. M., González-Gallego, J. \& Tuñón, M. J. $\mathrm{N}$-acetyl-cysteine protects liver from apoptotic death in an animal model of fulminant hepatic failure. Apoptosis 11, 1945-1957 (2006).

57. Crespo, I. et al. Melatonin prevents the decreased activity of antioxidant enzymes and activates nuclear erythroid 2-related factor 2 signaling in an animal model of fulminant hepatic failure of viral origin. J. Pineal Res. 49 193-200 (2010).

58. Crespo, I. et al. Melatonin inhibits the sphingosine kinase 1/sphingosine-1 phosphate signaling pathway in rabbits with fulminant hepatitis of viral origin. J. Pineal Res. 61, 168-176 (2016).

59. Le Gall-Reculé, G. et al. Emergence of a new lagovirus related to rabbit hemorrhagic disease virus. Vet. Res. 44, 81 (2013).

60. Nichols, H. J. \& Hough, W. H. Demonstration of Spirochaeta pallida in the cerebrospinal fluid. JAMA 60, 108-110 (1913)

61. Magnuson, H. J., Eagle, H. \& Fleischman, R. The minimal infectious inoculum of Spirochaeta pallida (Nichols Strain), and a consideration of its rate of multiplication in vivo. Am. J. Syph. 32, 1-18 (1948).

62. Turner, T. B. \& Hollander, D. H. Biology of the Treponematoses.. (World Health Organization, Geneva, Switzerland, 1957)

63. Sell, S. \& Norris, S. J. The biology, pathology, and immunology of syphilis. Int. Rev. Exp. Pathol. 24, 203-276 (1983).

64. Lithgow, K. V. et al. A defined syphilis vaccine candidate inhibits dissemination of Treponema pallidum subspecies pallidum. Nat. Commun. 8 14273 (2017)

65. Fitzgerald, T. J. Experimental congenital syphilis in rabbits. Can. J. Microbiol. 31, 757-762 (1985).

66. Froberg, M. K., Fitzgerald, T. J., Hamilton, T. R., Hamilton, B. \& Zarabi, M. Pathology of congenital syphilis in rabbits. Infect. Immun. 61, 4743-4749 (1993).

67. Tantalo, L. C., Lukehart, S. A. \& Marra, C. M. Treponema pallidum strain-specific differences in neuroinvasion and clinical phenotype in a rabbit model. J. Infect. Dis. 191, 75-80 (2005).

68. Schell, R. F., LeFrock, J. L., Chan, J. K. \& Bagasra, O. LSH hamster model of syphilitic infection. Infect. Immun. 28, 909-913 (1980).

69. Wicher, K., Miller, J. N., Urquhart, A. W. \& Wicher, V. Treponema pallidumimmobilizing antibodies in guinea pig experimental syphilis. Infect. Immun. 57, 2900-2902 (1989)

70. Gueft, B. \& Rosahn, P. D. Experimental mouse syphilis, a critical review of the literature. Am. J. Syph. 32, 59-88 (1948).

71. Giacani, L. et al. Antigenic variation in Treponema pallidum: Tprk sequence diversity accumulates in response to immune pressure during experimental syphilis. J. Immunol. 184, 3822-3829 (2010).

72. Molini, B. J. et al. Macrolide resistance in Treponema pallidum correlates with $23 \mathrm{~S}$ rDNA mutations in recently isolated clinical strains. Sex. Transm. Dis. $\mathbf{4 3}$ 579-583 (2016).

73. Cameron, C. E. \& Lukehart, S. A. Current status of syphilis vaccine development: need, challenges, prospects. Vaccine 32, 1602-1609 (2014).

74. Koch, R. Die aetiologie der tuberkulose. Berl. Klin. Wochenschr. 19, 221-230 (1882)

75. Lurie, M. B. Resistance to tuberculosis: Experimental studies in native and acquired defensive mechanisms. (Harvard University Press, Cambridge, MA, USA, 1964).

76. Mendez, S. et al. Susceptibility to tuberculosis: composition of tuberculous granulomas in Thorbecke and outbred New Zealand White rabbits. Vet. Immunol. Immunopathol. 122, 167-174 (2008).
77. Kaplan, G. \& Tsenova, L. Pulmonary tuberculosis in the rabbit. In: F. J. Leong, V. Dartois, T. Dick (eds). A Colour Atlas of comparative pulmonary tuberculosis histopathology. pp. 107-130. (CRC Press, Boca Raton, FL, USA, 2010).

78. Flynn, J. L., Tsenova, L., Izzo, A. \& Kaplan, G. Experimental animal models of tuberculosis. In: S. H. E. Kaufmann, W. J. Britton (eds). Handbook of tuberculosis. pp. 389-426. (Wiley-VCH Verlag GmbH \& Co, Weinheim, Germany, 2008).

79. Manabe, Y. C. et al. The aerosol rabbit model of TB latency, reactivation and immune reconstitution inflammatory syndrome. Tuberculosis 88, 187-196 (2008).

80. Liu, X. et al. Establishment of a rabbit model of spinal tuberculosis using Mycobacterium tuberculosis strain H37Rv. Jpn. J. Infect. Dis. 68, 89-97 (2015).

81. Subbian, S., Karakousis, P. \& Kaplan, G. Rabbit model of mycobacterial diseases. In: H. Mukundan, M. A. Chambers, R. W. Waters, M. H. Larsen (eds). Tuberculosis, leprosy and mycobacterial diseases of man and animals: The many hosts of mycobacteria. pp. 402-418. (CABI, Oxfordshire, UK, 2015).

82. Tsenova, L. et al. BCG vaccination confers poor protection against $M$. tuberculosis HN878-induced central nervous system disease. Vaccine $\mathbf{2 5}$ 5126-5132 (2007).

83. Dannenberg, A. J. Rabbit model of tuberculosis. In: B. R. Bloom (ed). Tuberculosis: pathogenesis, protection and control. pp. 149-156. (ASM Press, Washington, DC, USA, 1994).

84. Helke, K. L., Mankowski, J. L. \& Manabe, Y. C. Animal models of cavitation in pulmonary tuberculosis. Tuberculosis $\mathbf{8 6}, 337-348$ (2006).

85. Via, L. E. et al. Tuberculous granulomas are hypoxic in guinea pigs, rabbits, and nonhuman primates. Infect. Immun. 76, 2333-2340 (2008).

86. Manabe, Y. C. et al. Different strains of Mycobacterium tuberculosis cause various spectrums of disease in the rabbit model of tuberculosis. Infect. Immun. 71, 6004-6011 (2003)

87. Via, L. E. et al. Infection dynamics and response to chemotherapy in a rabbit model of tuberculosis using [(1)(8)F]2-fluoro-deoxy-D-glucose positron emission tomography and computed tomography. Antimicrob. Agents Chemother. 56, 4391-4402 (2012)

88. Kjellsson, M. C. et al. Pharmacokinetic evaluation of the penetration of antituberculosis agents in rabbit pulmonary lesions. Antimicrob. Agents Che mother. 56, 446-457 (2012).

89. Liu, P., Jiang, H., Li, S., Lin, Z. \& Jiang, J. Determination of anti-tuberculosis drug concentration and distribution from sustained release microspheres in the vertebrae of a spinal tuberculosis rabbit model. J. Orthop. Res. 35, 200-208 (2017).

90. Prideaux, B. et al. The association between sterilizing activity and drug distribution into tuberculosis. Nat. Med. 21, 1223-1227 (2015).

91. Zumla, A. et al. Host-directed therapies for infectious diseases: current status, recent progress, and future prospects. Lancet Infect. Dis. 16, e47-e63 (2016)

92. Subbian, S. et al. Phosphodiesterase-4 inhibition combined with isoniazid treatment of rabbits with pulmonary tuberculosis reduces macrophage activation and lung pathology. Am. J. Pathol. 179, 289-301 (2011).

93. Datta, M. et al. Anti-vascular endothelial growth factor treatment normalizes tuberculosis granuloma vasculature and improves small molecule delivery. Proc. Natl Acad. Sci. USA 112, 1827-1832 (2015).

94. Dannenberg, A. M. Jr. Perspectives on clinical and preclinical testing of new tuberculosis vaccines. Clin. Microbiol. Rev. 23, 781-794 (2010).

95. Converse, P. J. et al. Pulmonary bovine-type tuberculosis in rabbits: bacillary virulence, inhaled dose effects, tuberculin sensitivity, and Mycobacterium vaccae immunotherapy. Clin. Diagn. Lab. Immunol. 5, 871-881 (1998).

96. Breitburd, F. et al. Immunization with virus like particles from cottontail rabbit papillomavirus (CRPV) can protect against experimental CRPV infection. J. Virol. 69, 3959-3963 (1995).

97. Christensen, N. D. Cottontail rabbit papillomavirus (CRPV) model system to test antiviral and immunotherapeutic strategies. Antivir. Chem. Chemother. 16, 355-362 (2005).

98. Hu, J., Cladel, N. M., Balogh, K., Budgeon, L. \& Christensen, N. D. Impact of genetic changes to the CRPV genome and their application to the study of pathogenesis in vivo. Virology 358, 384-390 (2007).

99. Maglennon, G. A., Mclntosh, P. \& Doorbar, J. Persistence of viral DNA in the epithelial basal layer suggests a model for papillomavirus latency following immune regression. Virology 414, 153-163 (2011).

100. $\mathrm{Hu}$, J. et al. An HLA-A2.1-transgenic rabbit model to study immunity to papillomavirus infection. J. Immunol. 177, 8037-8045 (2006).

101. Bieniasz, P. D. \& Cullen, B. R. Multiple blocks to human immunodeficiency virus type 1 replication in rodent cells. J. Virol. 74, 9868-9877 (2000). 
102. Cohen, J. Building a small-animal model for AIDS, block by block. Science $\mathbf{2 9 3}$ 1034-1036 (2001).

103. Keppler, O. T. et al. Progress toward a human CD4/CCR5 transgenic rat mode for de novo infection by human immunodeficiency virus type 1. J. Exp. Med. 195, 719-736 (2002).

104. Michel, N. et al. Human cyclin T1 expression ameliorates a T-cell-specific transcriptional limitation for HIV in transgenic rats, but is not sufficient for a spreading infection of prototypic R5 HIV-1 strains ex vivo. Retrovirology 6, 2 (2009).

105. Cutino-Moguel, T. \& Fassati, A. A phenotypic recessive, post-entry block in rabbit cells that results in aberrant trafficking of HIV-1. Traffic 7, 978-992 (2006)

106. Schaller, T., Hue, S. \& Towers, G. J. An active TRIM5 protein in rabbits indicates a common antiviral ancestor for mammalian TRIM5 proteins. J. Virol. 81, 11713-11721 (2007).

107. Tervo, H. M. \& Keppler, O. T. High natural permissivity of primary rabbit cells for HIV-1, with a virion infectivity defect in macrophages as the final replication barrier. J. Virol. 84, 12300-12314 (2010).

108. Goffinet, C. et al. HIV-1 antagonism of CD317 is species specific and involves Vpu-mediated proteasomal degradation of the restriction factor. Cell Host Microbe 5, 285-297 (2009).

109. Ikeda, T. et al. The antiretroviral potency of APOBEC1 deaminase from small animal species. Nucleic Acids Res. 36, 6859-6871 (2008).

110. Zhang, J. X., Diehl, G. E. \& Littman, D. R. Relief of preintegration inhibition and characterization of additional blocks for HIV replication in primary mouse T cells. PLOS ONE 3, e2035 (2008).

111. Speck, R. F. et al. Rabbit cells expressing human CD4 and human CCR5 are highly permissive for human immunodeficiency virus type 1 infection. J. Virol. 72, 5728-5734 (1998)

112. Guo, R. et al. Generation and evaluation of Myostatin knock-out rabbits and goats using CRISPR/Cas9 system. Sci. Rep. 6, 29855 (2016).

113. Yang, D. et al. Identification and characterization of rabbit ROSA26 for gene knock-in and stable reporter gene expression. Sci. Rep. 6, 25161 (2016).

114. Torrents de la Peña, A. et al. Immunogenicity in rabbits of SOSIP trimers from clades A, B and C, given individually, sequentially or in combinations. J. Virol. 92, JVI.01957-17 (2018)

115. Sanders, R. W. et al. HIV-1 VACCINES. HIV-1 neutralizing antibodies induced by native-like envelope trimers. Science $\mathbf{3 4 9}$, aac4223 (2015).

116. Beddows, S. et al. A comparative immunogenicity study in rabbits of disulfide-stabilized, proteolytically cleaved, soluble trimeric human immunodeficiency virus type 1gp140, trimeric cleavage-defective gp140 and monomeric gp120. Virology 360, 329-340 (2007).

117. Zhang, P. F. et al. Extensively cross-reactive anti-HIV-1 neutralizing antibodies induced by gp140 immunization. Proc. Natl Acad. Sci. Usa. 104, 10193-10198 (2007)

118. Dong, M. et al. Induction of primary virus-cross-reactive human immunodeficiency virus type 1-neutralizing antibodies in small animals by using an alphavirus-derived in vivo expression system. J. Virol. 77, 3119-3130 (2003)

119. Kulp, D. W. et al. Structure-based design of native-like HIV-1 envelope trimers to silence non-neutralizing epitopes and eliminate CD4 binding. Nat. Commun. 8, 1655 (2017).

120. Law, M., Cardoso, R. M., Wilson, I. A. \& Burton, D. R. Antigenic and immunogenic study of membrane-proximal external region-grafted gp120 antigens by a DNA prime-protein boost immunization strategy. J. Virol. 81, 4272-4285 (2007).

121. Richmond, J. F. L. et al. Screening of HIV-1 Env glycoproteins for the ability to raise neutralizing antibody using DNA immunization and recombinant vaccinia virus boosting. Virology 230, 265-274 (1997).

122. Lu, S. et al. Immunogenicity of DNA vaccines expressing human immunodeficiency virus type-1 envelope glycoprotein with and without deletions in the V1/2 and V3 regions. AIDS Res. Hum. Retrovir. 14, 151-155 (1998).

123. Wang, S. et al. Enhanced immunogenicity of gp120 protein when combined with recombinant DNA priming to generate antibodies that neutralize the JR-FL primary isolate of human immunodeficiency virus type 1. J. Virol. 79 7933-7937 (2005).
124. Vaine, M. et al. Improved induction of antibodies against key neutralizing epitopes by human immunodeficiency virus type 1 gp120 DNA primeprotein boost vaccination compared to gp120 protein-only vaccination. J. Virol. 82, 7369-7378 (2008).

125. Vaine, M., Wang, S., Hackett, A., Arthos, J. \& Lu, S. Antibody responses elicited through homologous or heterologous prime-boost DNA and protein vaccinations differ in functional activity and avidity. Vaccine 28, 2999-3007 (2010).

126. Vaine, M. et al. Two closely related Env antigens from the same patient elicited different spectra of neutralizing antibodies against heterologous HIV-1 isolates. J. Virol. 85, 4927-4936 (2011).

127. Chen, Y. et al. A novel rabbit monoclonal antibody platform to dissect the diverse repertoire of antibody epitopes for HIV-1 Env immunogen design. J. Virol. 87, 10232-10243 (2013).

128. Pan, R. et al. Rabbit anti-HIV-1 monoclonal antibodies raised by immunization can mimic the antigen-binding modes of antibodies derived from HIV-1 infected humans. J. Virol. 87, 10221-10231 (2013).

129. Pan, R. et al. Structural analysis of a novel rabbit monoclonal antibody R53 targeting an epitope in HIV-1 gp120 C4 region critical for receptor and co-receptor binding. Emerg. Microb. Infect. 4, e44 (2015).

130. Liu, S., Wang, S. \& Lu, S. DNA immunization as a technology platform for monoclonal antibody induction. Emerg. Microb. Infect. 5, e33 (2016).

131. Srivastava, R. et al. Human asymptomatic epitopes identified from the herpes simplex virus tegument protein vp13/14 (ul47) preferentially recall polyfunctional effector memory cd44high cd62llow cd8+ tem cells and protect humanized hla-a*02:01 transgenic mice against ocular herpesvirus infection. J. Virol. 91, e01793-16 (2017).

132. Srivastava, R. et al. CXCL10/CXCR3-dependent mobilization of hsv-specific cd8+ tem and cd8+ trm cells within infected tissues allows efficient protection against recurrent herpes infection and disease. J. Virol. 91, e00278-17 (2017).

133. Nesburn, A. B. \& BenMohamed, L. A Tribute to Professor Steven L. Wechsler (1948-2016): The man and the scientist. Curr. Eye Res. 42, 161-162 (2017).

134. Nalbandian, A. et al. Activation of the NLRP3 inflammasome is associated with valosin-containing protein myopathy. Inflammation 40, 21-41 (2017).

135. Khan, A. A. et al. Bolstering the number and function of HSV-1-specific CD8+ effector memory $T$ cells and tissue-resident memory $T$ cells in latently infected trigeminal ganglia reduces recurrent ocular herpes infection and disease. J. Immunol. 199, 186-203 (2017).

136. Dasgupta, G. \& BenMohamed, L. Of mice and not humans: how reliable are animal models for evaluation of herpes CD8(+)-T cell-epitopes-based immunotherapeutic vaccine candidates? Vaccine 29, 5824-5836 (2011).

137. Chentoufi, A. A. et al. A novel HLA (HLA-A*0201) transgenic rabbit model for preclinical evaluation of human CD8+ T cell epitope-based vaccines against ocular herpes. J. Immunol. 184, 2561-2571 (2010).

138. Srivastava, R. et al. The herpes simplex virus latency-associated transcript gene is associated with a broader repertoire of virus-specific exhausted CD8 $+T$ cells retained within the trigeminal ganglia of latently infected hla transgenic rabbits. J. Virol. 90, 3913-3928 (2016).

139. Perng, G. C. et al. Large amounts of reactivated virus in tears precedes recurrent herpes stromal keratitis in stressed rabbits latently infected with herpes simplex virus. Curr. Eye Res. 41, 284-291 (2016).

140. Jester, J. V. et al. Confocal microscopic analysis of a rabbit eye model of highincidence recurrent herpes stromal keratitis. Cornea 35, 81-88 (2016).

141. Srivastava, R. et al. Herpes simplex virus type 1 human asymptomatic CD8 T cell epitopes protect against ocular herpes in "humanized" HLA transgenic rabbit model. IOVS 194, 2232-2248 (2015).

142. Srivastava, R. et al. A herpes simplex virus type 1 human asymptomatic CD8+ T-cell epitopes-based vaccine protects against ocular herpes in a "humanized" HLA transgenic rabbit model. Invest. Ophthalmol. Vis. Sci. $\mathbf{5 6}$ 4013-4028 (2015).

143. Khan, A. A. et al. Therapeutic immunization with a mixture of herpes simplex virus 1 glycoprotein D-derived "asymptomatic" human CD8+ T-cell epitopes decreases spontaneous ocular shedding in latently infected HLA transgenic rabbits: association with low frequency of local PD-1+ TIM-3+ CD8+ exhausted T cells. J. Virol. 89, 6619-6632 (2015). 